\title{
Design and Analysis of Frequency Selective Surface at Ka/K/Ku Band
}

\section{${ }^{1}$ Kirti Chandel \\ ${ }^{2}$ Seema Singh}

1,2 MTech Student ICL Group of Colleges, Head of the Department ICL Group of Colleges.

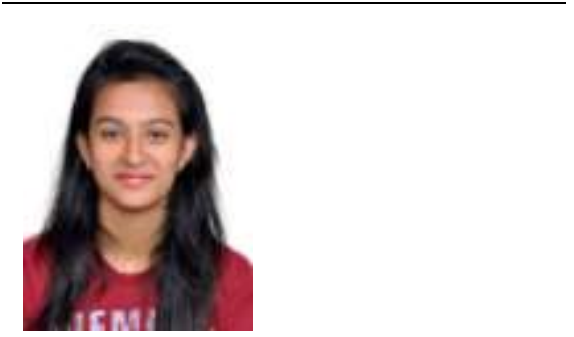

Corresponding author:

Kirti Chandel

chandel.kirti.singh93@gmail.com

Received: Sep 21, 2017

Revised: Sep 23, 2017

Published: Oct 31, 2017

\begin{abstract}
In this paper we will give a new way to designing spatial filters (frequency selective surfaces) being widely used in several microwave and optical systems. In this paper we will analyze transmission and reflection of electromagnetic waves from complex thick/thin metallic frequency selective surface (FSS). In this work, we have designed the spatial filter (FSS) to be used in microwave/satellite communication. We have tried to overcome the limitations in form of accuracy and computational effort. Using CST software, the results for full wave analysis method are being evaluated.
\end{abstract}

Keywords- Frequency Selective Surface (FSS), Microwave Spectrum, CST, Double Square Loop FSS, Gain, Directivity, Efficiency. 


\section{INTRODUCTION}

Frequency Selective Surface (FSS) are periodic structures with a band-pass or a band-stop frequency response. FSS are planar periodic structures of identical patches or apertures of conducting elements repeating periodically in either a one or two- dimensional array of a dielectric substrate (Munk, B.A., 2000; Wu, T.K., 1995; Lui et al., 2007; Mias et al., 2001; Sakran and Neve-Oz, 2008). Because of their frequency selective properties, FSS are incorporated in a wide variety of applications such as the realization of reflector antennas, random design, making polarizers and beam splitters, and also as radar absorbing structure (Lui et al., 2007). In this paper we will discuss the Frequency Selective Surface as a microwave filter. A microwave filter is a two-port network used to control the frequency response at certain point in a microwave system by providing transmission at frequencies within the pass-band of the filter and attenuation in the stop-band of the filter (Pozar, 2004). We will focus mainly on $\mathrm{Ku} / \mathrm{K} / \mathrm{Ka}$ bands $(12-40 \mathrm{GHz})$. The Earth atmosphere has different behavior for different frequency range. So taking all the factors into account we design our spatial filters (FSS). There is long history of development of Frequency Selective Surface Technology. Over the past few decades, FSSs have found numerous applications in both the commercial and military sectors to provide multiple frequency band operation. Screen door of a microwave oven is the one that we can see in our daily life. It is designed to reflect microwave energies consisting of periodic array at $2.45 \mathrm{GHz}$ while allowing light to pass through.

Frequency Selective Surface (FSS) is a group of intermittent elements (unit cell). These can be each of two slots on the regulating sheet or metallic patches on subtracts. When an EM (electromagnetic) wave is scene on the surface, every aspect (unit cell) reverberates and demobilizes the energy about its sonority frequency Pozar, 2004). The circumstance EM wave is partly transmits through the structure and slightly reflected. These were said to be a passive array. The metallic plot on the substrate FSS properties are revered as capacitive FSS. They can also operate like low pass filter.

FSS can be categorized as four types. Centre connected array of elements oscillates when the finest tip to tip is generally same as one half of the acumen. The aspects must be closely spaced for the outcome of better output. The loop structure resonates when their average width is equal to half wavelength and this can be worn for moisturizing and these might be decreased to three tenth of the wavelength without any substrate. Therefore by adopting this we can deed precise bandwidth to sensational wide bandwidth. Study interior types should also be one half the wavelengths and also add many alternative factors. The merger types have many considerations and there are a large statistics of designs placed on this usually adopted to contour the resonant curves to the needs of the designer since they allows them beyond many restraints, still reckoning the curve are equally tougher. Therefore many tweaks will be required in the tougher case. The advantage of the single-layer FSSs having the dual-resonant elements is that they are light in weight and compact in volume, compared with the multilayered FSSs. The structures proposed so far are an array of two different double square loops (Wu, 1994 and Chuprin, 2000), length dipoles (Hill and Munk, 1996), concentric rings (Wu and Lee, 1994 and Huang, 1994), and so on.

\section{LITERATURE REVIEW}

Frequency Selective Surface (FSS) is a periodic array of metallic patches or its complement metallic apertures, etched on a dielectric substrate, which acts as a spatial filter (cst.com, 2016). Filter characteristics of the FSS are mainly dependent on the surface's element pattern. 


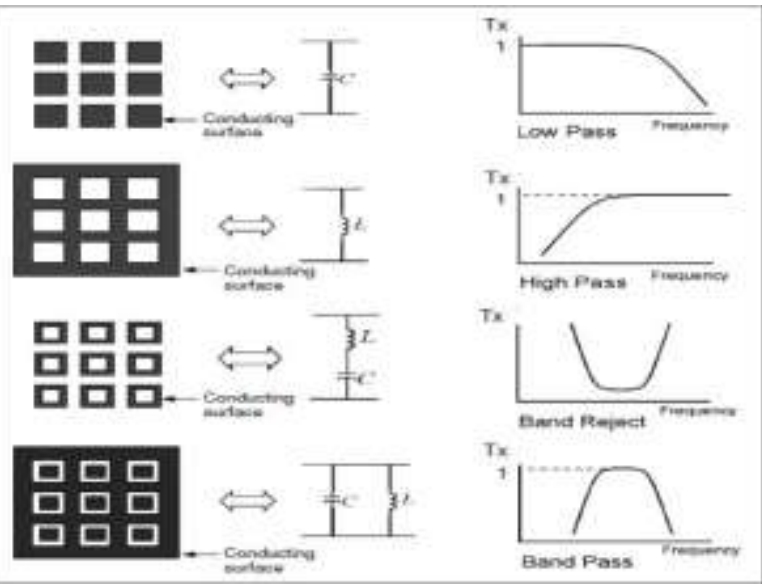

Fig-1: Filter responses of the FSS [28]

Thus, by adjusting the pattern of the element, FSS can be designed as a low-pass, high-pass, band-pass or band-stop filters, as shown in the Figure1.The patch element group consists of both band-stop and low-pass FSSs, whereas band-pass and high-pass FSSs belong to the aperture or slot group.

Depending on the applications, there are cases where the signals need to be confined or amplified. For instance, a band-pass FSS is deposited on the energy-saving glass to overcome its limitation.

\section{FSS Element Geometry:}

One of the important factors that can influence the frequency response of the FSS is the FSS element geometry. FSS element is not only limited to square shape, but it is also can be designed as circular, hexagon or more convoluted shapes.

In general, FSS has four major groups of elementtype, which are centre connected, loop types, solid interior or plate types and combinations. Each group of elements offers different performances in terms of the angular stability, cross-polarization, band width and band separation. Therefore, it is very crucial to choose the FSS element that meets all the requirements needed for that particular application.

In terms of the bandwidth, the first group, which is the centre connected elements such as dipoles, tripoles, Jerusalem crosses and cross dipoles are categorized as the most narrow-banded elements.
On the other hand, the second group which is the loop elements, for instance, square loop, hexagon loop and circular loop generally offer a good bandwidth performance. Since loop elements mostly have small $\mathrm{x}$ and $\mathrm{y}$ dimensions, it can be spaced close to one another. Therefore, this type of element is very suitable to design a multi-band FSS.

On the other hand, solid interior or plate elements have undesirable characteristics. First, it is very hard to achieve resonance for this type of elements, as they are highly inductive elements with small capacitances between them. Second, these elements cannot be placed close to one another as their $\mathrm{x}$ - and $y$ - dimensions are around a half of a wavelength, which prohibit them to have more than one band.

\section{Conductivity of the Element:}

It is very essential to use the FSS element with high conductivity in order to ensure that the designed FSS is capable to tune the target resonance frequency. This is due to the fact that the element with low conductivity will degrade the FSS performances. Besides, if the material with extremely small conductivity is used as the FSS element, the structure will no longer behave as a frequency selective but it will act like a common dielectric plate.

In addition, the conductive material needs to be selected properly as different fabrication techniques are required if different conductive materials are used. In addition, the conductive material needs to be selected properly as different fabrication techniques are required if different conductive materials are used.

The most common fabrication technique that has been widely used is the photolithography technique, which involves multiple steps such as film laminating, UV exposure, developing, etching and stripping. One of the drawbacks of this technique is that the choice of the substrates is limited since the solvent used in the etching process is corrosive. Usually, copper or aluminium will be used as the conductive material as the price is reasonable.

An alternative of the above-mentioned conventional fabrication technique is the printing technique 
which can be performed by utilizing the inkjet printer and conductive ink. This technique is more environmental friendly, it can reduce high volumes of chemical waste generated through the photolithography process.

In our work we have chosen Double Square Loop structure of FSS to get Band-Pass frequency response. Double Square Loop holds an edge on other structures due to its advantages such as simple geometry, easy fabrication, high selectivity and multi-band application. Modification of this FSS structure is easily achieved and it is normally, insensitive to the change in the polarization and the angle of incidence. In our work we have used this Double Square Loop Structure to get band pass filter response at different bands of the microwave spectrum.

\section{LIMITATION OF THE PRESENT APPROACH}

Earlier many methods have been adopted to analyze the reflection and transmission characteristics of a FSS, such as Method of Moment (MOM), Finite Element Method (FEM), Finite Difference Time Domain Method (FDTD), Equivalent Circuit Model, Full Wave Analysis Method and spectral domain approach etc. Although from above methods we can analyze our simulation approach but how can we validate our design, means there should be some method to validate our results.

\section{DESIGN AND SIMULATION}

A frequency-selective surface (FSS) is a periodic, planar assembly of generally metallic elements on a dielectric layer. It has recently been widely used in variety of electromagnetic applications, such as radomes (terrestrial and airborne), electromagnetic shielding, absorbers and antennas. Unlike traditional microwave filters, the frequency response of FSS is not only functions of frequency, but also functions of incident of angle and polarizations of EM waves.

We present the analysis and modeling of detailed investigation and study of double square loop elements in FSS. The simulations of such elements are performed with full wave simulation tool CST Microwave Studio on single substrate.

\section{Goal of designs:}

The circuit will be designed using micro strip technology on printed circuit board that can be mounted on a wall, andwire net that canbe built in open areas.

\section{Simulation Software CST}

The designs and simulations in this project are based on the Computer Simulation Technology (CST) MicrowaveStudio Suitewhich is ahigh performance electromagnetic simulation software.There are two basic solver modules provided: time domain solver and frequency domain solver. The two solvers are totally different. Time domain solver is used for non-resonant structures and frequency domain solver contains alternatives for highly resonant structures .Beside frequency domainsolver has theoption of utilizing tetrahedral mesh that can discrete the structure better whichis not availablewith in timedomain solver .In addition, time domain solver is only fornormal incidence butfrequency domain solvercan be used for off -normal incidences. Because ofthe aboveI used the frequencydomain solver tosimulate designs.

\section{Basics of the Designs:}

Designs are mainly based on the shapes of double square loop to achieve the desired resonances.

\section{BAND-PASS DOUBLE SQUARE LOOP FSS:}

\section{Structure of FSS:}

The unit cell dimensions of band-stop FSSare shown inFig-2. The band-stop characteristics are achieved by designingtwo square-loop elements of different dimensions on FR-4 substrate. The thickness of FR-4 substrate is $1.6 \mathrm{~mm}$ and relative permittivity ( ) andthe loss tangent $(\tan \delta$ ) are 4.3 and 0.02 , respectively. The circumference of the outer square-loop elementis $20 \mathrm{~mm}$ while the 
circumference of the inner square loop element is $16 \mathrm{~mm}$. The width of outer square is $0.5 \mathrm{~mm}$ while the width of the inner square loop is $0.25 \mathrm{~mm}$. The dimensions of the FSS structure are depicted in Table1.

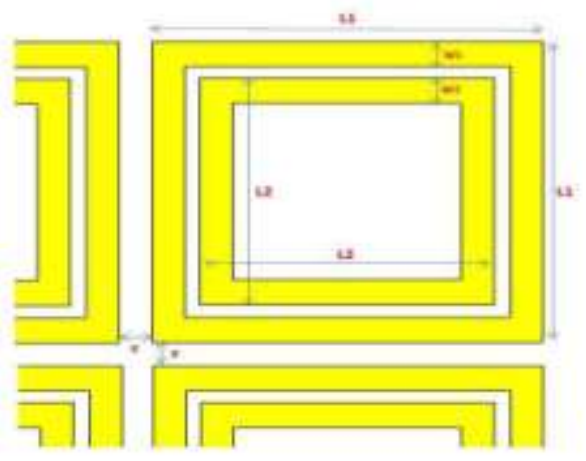

a) Top view

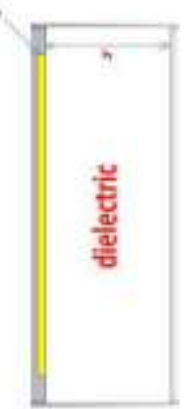

b) Side view
Fig-2: FSS structure Front and Side View a ( Dimensions ofSquare Loop FSS, b) Side View of the Model

Table-1: Dimensions of the Design

$\begin{array}{ccccccc}\text { Name } & \text { L1 } & \text { L2 } & \text { W1 } & \text { W2 } & \text { h } & \text { er } \\ \begin{array}{c}\text { Value } \\ (\mathrm{mm})\end{array} & 5 & 4 & 0.5 & 0.25 & 1.5 & 4.3\end{array}$

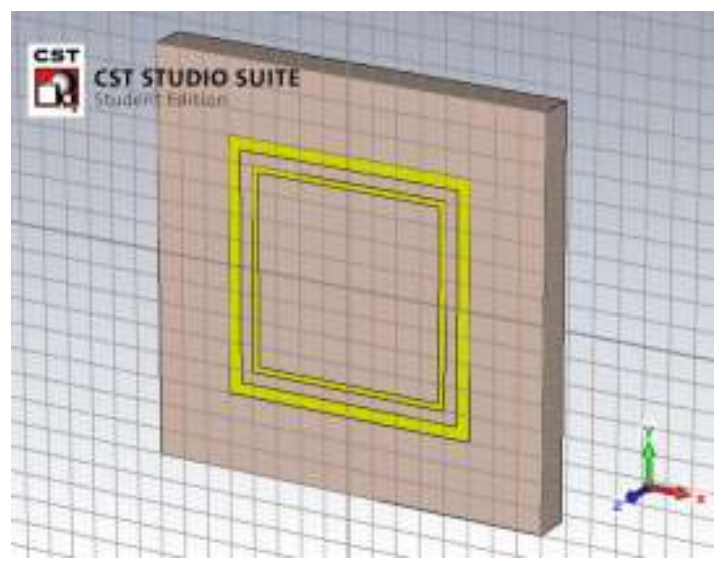

Fig-3: Double Square Loop FSS structure in CST

\section{SIMULATION RESULTS}

\section{FSS Parameters}

Directivity: The directivity or gain of an antenna is defined as the ratio of the maximum value of the power radiated per unit solid angle, to the average of total power radiation per unit solid angle: $\mathrm{G}=\{(\mathrm{dp} / \mathrm{d} \Omega) \max \} /(\mathrm{p} / 4 \pi)$

$S$ Parameters: S parameter is a parameter related to high frequency microwave circuits and denotes the properties of the network under high frequencies, reflection co-efficient and help in ports, the number of parameter involved is $\mathrm{n}^{\wedge} 2$.

Return loss $[\mathrm{db}]=-20 * \log (\mathrm{s} 11)$

S11 (Return loss): Return loss is defined as the destruction of the signal power resulted from the rumination caused at a discontinuity of transmission line or optical fibre. This discontinuity can be a mismatched with the help of load or a device.

$\mathrm{RL}(\mathrm{dB})=10 \log 10 \mathrm{pi} / \mathrm{pr}$

S21 (Forward Transmission Co-Efficient): It is the attenuation based on the wave travelling from port 1 to port 2 .

$\mathrm{S} 21=(\mathrm{b} 2 / \mathrm{a} 1)$

S12 (Forward Gain): It is attenuation of wave travelling from port 2 to port 1 .

$\mathrm{S} 12=(\mathrm{b} 1 / \mathrm{a} 1)$

S22 (Output Reflection Co-Efficient): Reflection coefficient illustrate either the amplitude of the signal or the ferocity of a reflected wave which is related to the incident wave. The reflection coefficient is related closely to the transmission coefficient.

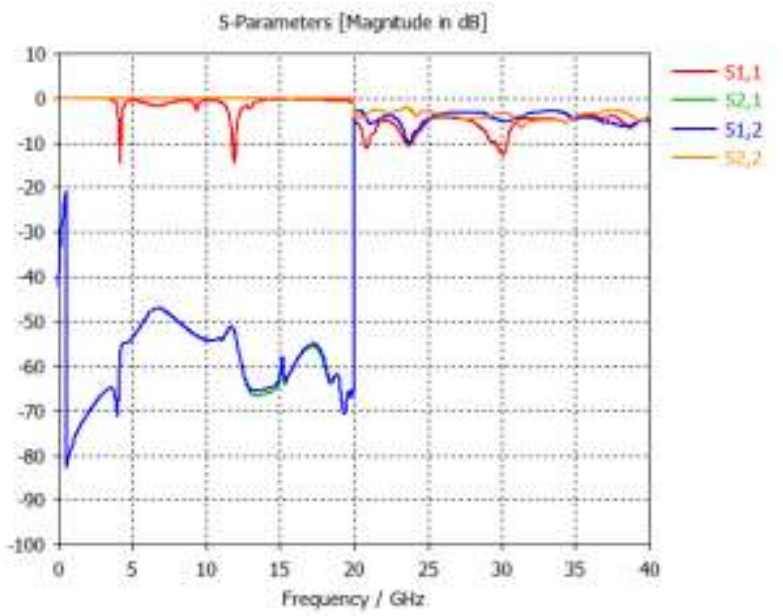

Fig-4: Graphical Representation of Results (S-Parameters) 


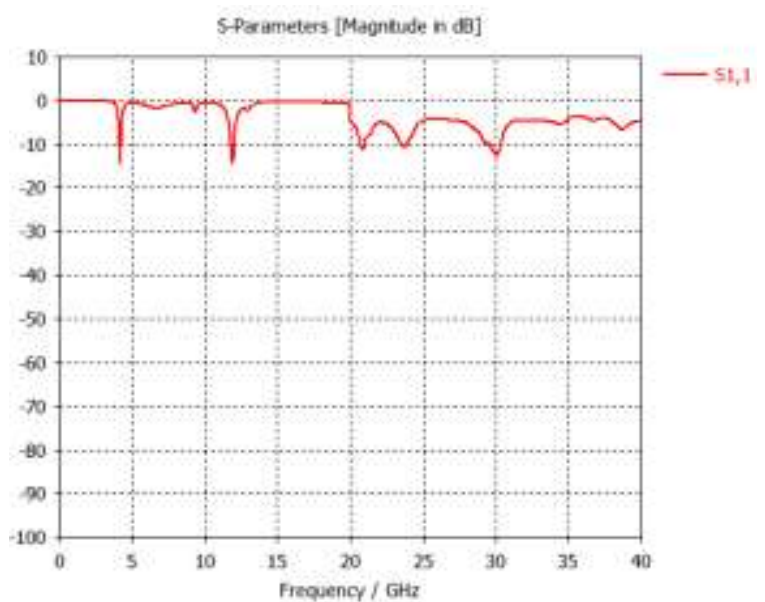

Fig-5: Graphical Representation of S11 Parameters

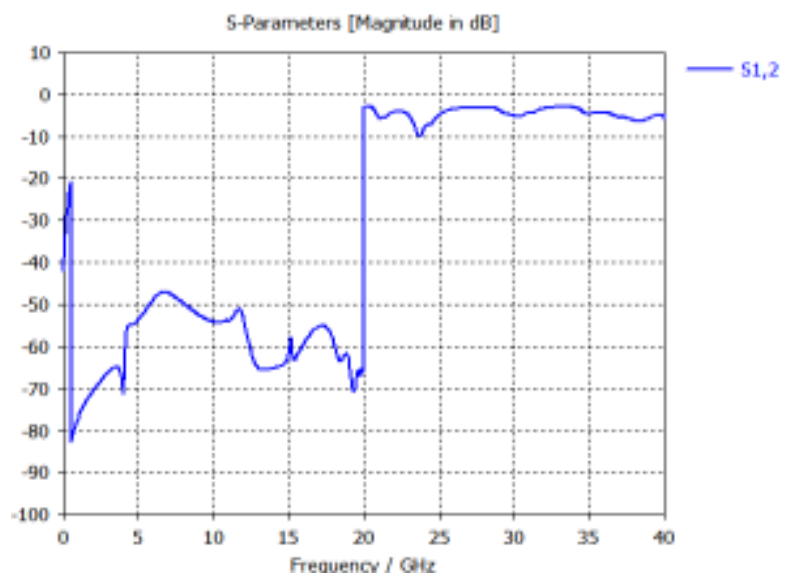

Fig-6: Graphical Representation of S12 Parameters

\section{CONCLUSION AND FUTURE SCOPES}

In this thesis, we have investigated a synthesis technique to yield the geometrical parameters of the Double square loop FSS, which is significantly useful for the fast analysis and design of FSS structure. The process of computation of different geometrical parameters is presented, and the parameters achieved by this synthesis technique are supported by the experimental as well as simulation results. At every frequency of interest, the numerical analysis is validated by comparing the resonance frequency with three different commercially available simulators based on different computational techniques. The proposed synthesis technique is used to design band-stop
Double square loop FSS at different frequencies (3 $\mathrm{GHz}, 22 \mathrm{GHz}$ and $26 \mathrm{GHz}$ ) and further extended to design the band-pass Double square loop FSS structure. Moreover, a way to control the reflection at any chosen frequency is discussed, which may find potential applications in controlling the reflection coefficient at various frequencies of the electromagnetic spectrum. The developed mathematical expression is significantly applicable for both types of free-standing Double square loop FSS structures such as patch-and slot-type. Furthermore, the dielectric substrate provides the mechanical strength, miniaturization and stable resonant frequency of the Double loop FSS structure. The significant implementation of developed mathematical expression of dielectric backed Double Square loop FSS on the square loop FSS yields novel modified square loop FSS structure.

We have considered the negligible thickness of the metallic pattern that is single square loop and circular ring $(\mathrm{t}<<\lambda)$, however it plays very important role over the circuit parameters and consequently opens the door for 3-dimensional FSS. However, various researchers are working over such structures using the substrate integrated waveguide (SIW), a stepped impedance resonator (SIR) and micro strip line. In addition to this, the design as well as fabrication issues of the conical surface FSS structure is also very important issue that will significantly improves the performance of the FSS structure. Moreover, the FSS structures provide significant applications in higher frequency bands (terahertz) such as directivity/gain enhancement in antenna systems, sensing and imaging. Therefore, it is required to exploit the FSS structure in the terahertz regime of the electromagnetic spectrum and various issues such as losses, surface roughness and skin depth need to be investigated in detail while investigating FSS structures in the terahertz regime. 


\section{REFERENCES}

1. Munk, B.A., (2000). Frequency Selective Surfaces- Theory and Design, John Wiley and Sons, Inc., New York.

2. Wu, T.K., (1995). Frequency Selective Surfaces and Grid Array, John Wiley and Sons, Inc., New York.

3. Lui, H.T., H. F. Cheng, Z.Y. Chu, et al., (2007). Absorbing properties of frequency selective surface absorbers with cross-shaped resistive patches, Material Design, 28: 21662171.

4. Mias, C., C. Tsakonas, and C. Oswald, (2001). An investigation into the feasibility of designing frequency selective windows employing periodic structures, (Ref. AY3922), Tech. Rep., Final Report for the Radio-communications Agency, Nottingham Trent University.

5. Sakran, F. and Y. Neve-Oz, (2008). Absorbing frequency-selective-surface for the mm- wave range, IEEE Transactions on Antennas and Propagation, 56(8): 2649-2655.

6. David M. Pozar, (2004). Microwave Engineering (Third Edition), Wiley.
7. T. K. Wu, (1994). Four-band frequency selective surface with double-square-loop patch elements, IEEE Trans. Antennas Propagat., 42: 1659-1663.

8. A. D. Chuprin, E. A. Parker, and J. C. Batchelor, (2000). Convoluted double square: Single layer FSS with close band spacings," Electron. Lett., 36: 1830-1831.

9. R. A. Hill and B. A. Munk, (1996). The effect of perturbating a frequency selec-tive surface and its relation to the design of a dual-band surface, IEEE Trans. Antennas Propagat., 44: $368-374$.

10. T. K. Wu and S. W. Lee, (1994). Multiband frequency selective surface with multiring patch elements," IEEE Trans. Antennas Propagat., 42: 1484-1490.

11. J. Huang, T. K. Wu, and S. H. Lee, (1994). Triband frequency selective surface with circular ring elements," IEEE Trans. Antennas Propagat., 42: 166-175.

12. Cst.com, "CST - Computer Simulation Technology", 2016. [Online]. Available: https://www.cst.com/. [Accessed: 08- Jan2016]. 